\title{
Fixed point theorems for generalized almost contractions in partial metric spaces
}

\author{
Ishak Altun · Kishin Sadarangani
}

Received: 10 February 2014/Accepted: 30 April 2014/Published online: 23 May 2014

(c) The Author(s) 2014. This article is published with open access at Springerlink.com

\begin{abstract}
In the present paper, we give some fixed point results for generalized Ćirić type strong almost contractions on partial metric spaces which generalizes some recent results appearing in the literature. Particularly, our result has as a particular case, mappings satisfying a general contractive condition of integral type.
\end{abstract}

Keywords Fixed point $\cdot$ Partial metric space $\cdot$ Almost contraction

\section{Mathematics Subject Classification (2000) Primary $54 \mathrm{H} 25 \cdot$ Secondary $47 \mathrm{H} 10$}

\section{Introduction}

Partial metric spaces were introduced by Matthews in [20] as a part of the study of denotational semantics of dataflow networks. These spaces are a generalization of usual metric spaces where the self distance for any point need not be equal to zero.

Let us recall that a partial metric on a set $X$ is a function $p: X \times X \rightarrow[0, \infty)$ such that for all $x, y, z \in X:$ (1) $x=$ $y \Longleftrightarrow p(x, x)=p(x, y)=p(y, y) \quad\left(T_{0}\right.$-separation axiom), (2) $p(x, x) \leq p(x, y) \quad$ (small self-distance axiom), (3) $p(x, y)=p(y, x) \quad$ (symmetry), (4) $\quad p(x, y) \leq p(x, z)+$ $p(z, y)-p(z, z)$ (modified triangular inequality).

\section{Altun $(\bowtie)$}

Department of Mathematics, Faculty of Science and Arts,

Kirikkale University, Yahsihan, 71540 Kirikkale, Turkey

e-mail: ishakaltun@yahoo.com

\section{K. Sadarangani}

Departamento de Matemáticas, Universidad de Las Palmas de

Gran Canaria, Campus de Tafira Baja,

35017 Las Palmas de Gran Canaria, Spain

e-mail: ksadaran@dma.ulpgc.es
A partial metric space (for short PMS) is a pair $(X, p)$ such that $X$ is a nonempty set and $p$ is a partial metric on $X$.

It is clear that, if $p(x, y)=0$, then $x=y$. But if $x=y$, $p(x, y)$ may not be 0 .

At this point it seems interesting to remark the fact that partial metric spaces play an important role in constructing models in the theory of computation (see for instance [1517], etc).

Example 1 Let $X=[0, \infty)$ and $p(x, y)=\max \{x, y\}$ for all $x, y \in X$. Then $(X, p)$ is a PMS.

Example 2 Let $I$ denote the set of all intervals $[a, b]$ for some real numbers $a \leq b$. Let $p: I \times I \rightarrow[0, \infty)$ be the function such that $p([a, b],[c, d])=\max \{b, d\}-\min \{a, c\}$. Then $(I, p)$ is a PMS.

Example 3 Let $X=\mathbb{R}$ and $p(x, y)=e^{\max \{x, y\}}$ for all $x, y \in X$. Then $(X, p)$ is a PMS.

Other examples of partial metric spaces may be found in [16, 18, 20, 22], etc.

Each partial metric $p$ on $X$ generates a $T_{0}$ topology $\tau_{p}$ on $X$ which has as a base the family open $p$-balls

$\left\{B_{p}(x, \varepsilon): x \in X, \varepsilon>0\right\}$,

where

$B_{p}(x, \varepsilon)=\{y \in X: p(x, y)<p(x, x)+\varepsilon\}$,

for all $x \in X$ and $\varepsilon>0$.

Observe that a sequence $\left\{x_{n}\right\}$ in a PMS $(X, p)$, converges to a point $x \in X$, with respect to $\tau_{p}$, if and only if $p(x, x)=\lim _{n \rightarrow \infty} p\left(x, x_{n}\right)$.

If $p$ is a partial metric on $X$, then the functions $p^{s}, p^{w}$ : $X \times X \rightarrow \mathbb{R}^{+}:=[0, \infty)$, given by 


$$
p^{s}(x, y)=2 p(x, y)-p(x, x)-p(y, y)
$$

and

$$
\begin{aligned}
p^{w}(x, y) & =\max \{p(x, y)-p(x, x), p(x, y)-p(y, y)\} \\
& =p(x, y)-\min \{p(x, x), p(y, y)\}
\end{aligned}
$$

are ordinary metrics on $X$. It is easy to see that $p^{s}$ and $p^{w}$ are equivalent metrics on $X$.

According to [20], a sequence $\left\{x_{n}\right\}$ in a PMS $(X, p)$ converges, with respect to $\tau_{p^{s}}$, to a point $x \in X$ if and only if

$\lim _{n, m \rightarrow \infty} p\left(x_{n}, x_{m}\right)=\lim _{n \rightarrow \infty} p\left(x_{n}, x\right)=p(x, x)$.

A sequence $\left\{x_{n}\right\}$ in a PMS $(X, p)$ is called a Cauchy sequence if $\lim _{n, m \rightarrow \infty} p\left(x_{n}, x_{m}\right)$ exists (and is finite). $(X, p)$ is called complete if every Cauchy sequence $\left\{x_{n}\right\}_{n \in \omega}$ in $X$ converges, with respect to $\tau_{p}$, to a point $x \in X$ such that $p(x, x)=\lim _{n, m \rightarrow \infty} p\left(x_{n}, x_{m}\right)$.

Finally, the following crucial facts are shown in [20]:

1. $\left\{x_{n}\right\}$ is a Cauchy sequence in $(X, p)$ if and only if it is a Cauchy sequence in the metric space $\left(X, p^{s}\right)$.

2. $(X, p)$ is complete if and only if $\left(X, p^{s}\right)$ is complete.

Matthews obtained, among other results, a partial metric version of the Banach fixed point theorem ([20, Theorem 5.3]) as follows.

Theorem 1 ([20]) Let $(X, p)$ be a complete partial metric space and let $T: X \rightarrow X$ be a contraction mapping, that is, there exists $\lambda \in[0,1)$ such that

$p(T x, T y) \leq \lambda p(x, y)$

for all $x, y \in X$. Then $T$ has a unique fixed point $z \in X$. Moreover, $p(z, z)=0$.

Later on, Abdeljawad et al. [1], Acar et al. [2, 3], Altun et al. [6-8], Karapinar and Erhan [19], Oltra and Valero [21] and Valero [27], gave some generalizations of the result of Matthews. Also, Cirić et al. [14], Samet et al. [25] and Shatanawi et al. [26] proved some common fixed point results in partial metric spaces. The best two generalizations of it were given by Romaguera [23, 24].

Theorem 2 Let $(X, p)$ be a complete partial metric space and let $T: X \rightarrow X$ be a map such that

$p(T x, T y) \leq \varphi(M(x, y))$

for all $x, y \in X$, where

$M(x, y)=\max \left\{p(x, y), p(x, T x), p(y, T y), \frac{1}{2}[p(x, T y)+p(y, T x)]\right\}$

and $\varphi$ satisfies one of the following:

1. $\varphi:[0, \infty) \rightarrow[0, \infty)$ is [23] an upper semicontinuous from the right such that $\varphi(t)<t$ for all $t>0$,
2. $\varphi:[0, \infty) \rightarrow[0, \infty)$ is a [24] nondecreasing function such that $\varphi^{n}(t) \rightarrow 0$ as $n \rightarrow \infty$ for all $t>0$.

Then $T$ has a unique fixed point $z \in X$. Moreover, $p(z, z)=0$.

In [22], Romaguera defined the 0-complete PMS as follows: A sequence $\left\{x_{n}\right\}$ in a PMS $(X, p)$ is called 0 Cauchy if

$\lim _{m, n \rightarrow \infty} p\left(x_{n}, x_{m}\right)=0$

and $(X, p)$ is called 0 -complete if every 0 -Cauchy sequence in $X$ converges, with respect to $\tau_{p}$, to a point $z \in X$ such that $p(z, z)=0$. It is clear that every complete PMS is 0 complete, but as it was shown in [22] the converse is not true.

On the other hand Berinde [9-11] defined weak contraction (or $(\delta, L)$-weak contraction) mappings in a metric space as follows.

Definition 1 Let $(X, d)$ be a metric space and $T: X \rightarrow X$ be a self operator. $T$ is said to be a weak contraction (or $(\delta, L)$-weak contraction) if there exists a constant $\delta \in(0,1)$ and some $L \geq 0$ such that

$d(T x, T y) \leq \delta d(x, y)+L d(y, T x)$

for all $x, y \in X$.

Note that, by the symmetry property of the distance, the weak contraction condition implicitly includes the following dual one

$d(T x, T y) \leq \delta d(x, y)+L d(x, T y)$

for all $x, y \in X$. So, in order to check the weak contractiveness of a mapping $T$, it is necessary to check both (1.2) and (1.3).

In [9] and [11], Berinde showed that any Banach, Kannan, Chatterjea and Zamfirescu mappings are weak contraction. Using the concept of weak contraction mappings, Berinde [9] proved that if $T$ is a $(\delta, L)$-weak contraction self mapping of a complete metric space $X$, then $T$ has a fixed point. Also, Berinde shows that any $(\delta, L)$-weak contraction mapping is a Picard operator. Then, Berinde [12] introduced the nonlinear type weak contraction using a comparison function and proved the following fixed point theorem. A map $\varphi: \mathbb{R}^{+} \rightarrow \mathbb{R}^{+}$, where $\mathbb{R}^{+}=[0, \infty)$, is called comparison function if it satisfies:

1. $\varphi$ is monotone increasing,

2. $\lim _{n \rightarrow \infty} \varphi^{n}(t)=0$ for all $t \in \mathbb{R}^{+}$.

If $\varphi$ satisfies (1) and

3. $\sum_{n=0}^{\infty} \varphi^{n}(t)$ converges for all $t \in \mathbb{R}^{+}$, then $\varphi$ is said to be $(c)$-comparison function. 
It is clear that $(c)$-comparison function implies comparison function, but the converse may not be true. We can find some properties and examples of comparison and $(c)$ comparison functions in [11].

Definition 2 Let $(X, d)$ be a metric space and $T: X \rightarrow X$ is a self operator. $T$ be said to be a weak $\varphi$-contraction (or $(\varphi, L)$-weak contraction) if there exists a comparison function $\varphi$ and some $L \geq 0$ such that

$$
d(T x, T y) \leq \varphi(d(x, y))+L d(y, T x)
$$

for all $x, y \in X$.

Similar to the case of weak contraction, in order to check the weak $\varphi$-contractiveness of a mapping $T$, it is necessary to check both (1.4) and

$d(T x, T y) \leq \varphi(d(x, y))+L d(x, T y)$

for all $x, y \in X$.

Clearly any weak contraction is a weak $\varphi$-contraction, but the converse may not be true. Also the class of weak $\varphi$-contractions includes Matkowski type nonlinear contractions.

Theorem 3 Let $(X, d)$ be a complete metric space and $T: X \rightarrow X$ be $(\varphi, L)$-weak contraction with $\varphi$ is $(c)$-comparison function. Then $T$ has a fixed point.

Let $(X, d)$ be a metric space and $T: X \rightarrow X$ be a map such that

$d(T x, T y) \leq \alpha M_{d}(x, y)+L d(y, T x)$

for all $x, y \in X$, where $\alpha \in[0,1), L \geq 0$,

$$
\begin{aligned}
& M_{d}(x, y) \\
& =\max \left\{d(x, y), d(x, T x), d(y, T y), \frac{1}{2}[d(x, T y)+p(y, T x)]\right\} .
\end{aligned}
$$

Then $T$ is called Ciric type strong almost contraction [13].

In light of the above information, Altun and Acar [5] introduced the concepts of weak and weak $\varphi$-contractions in the sense of Berinde on partial metric space, showed that any Banach, Kannan, Chatterjea and Zamfirescu mappings are weak contraction and proved some fixed point theorems in this interesting space.

Let $(X, p)$ be a partial metric space. A map $T: X \rightarrow X$ is called $(\varphi, L)$-weak contraction if there exists a comparison function $\varphi$ and some $L \geq 0$ such that

$p(T x, T y) \leq \varphi(p(x, y))+L p^{w}(y, T x)$

for all $x, y \in X$.

As above, because of the symmetry of the distance, the $(\varphi, L)$-weak contraction condition implicitly includes the following dual one

$p(T x, T y) \leq \varphi(p(x, y))+L p^{w}(x, T y)$ for all $x, y \in X$. Consequently, in order to check the $(\varphi, L)$ weak contractiveness of $T$, it is necessary to check both (1.6) and (1.7).

Theorem 4 Let $(X, p)$ be a 0 -complete partial metric space and $T: X \rightarrow X$ be $(\varphi, L)$ weak contraction with a (c)-comparison function. Then $T$ has a fixed point.

Later, Acar et al generalized Theorem 4 to Ciric type strong almost contractions and they proved the following results.

Theorem 5 Let $(X, p)$ be a 0-complete partial metric space and $T: X \rightarrow X$ be a map such that

$p(T x, T y) \leq \varphi(M(x, y))+L p^{w}(y, T x)$

for all $x, y \in X$, where $L \geq 0, \varphi$ is a $(c)$-comparison function and $M(x, y)$ as in Theorem 2 .

Then $T$ has a fixed point in $X$.

Theorem 6 Let $(X, p)$ be a 0-complete partial metric space and $T: X \rightarrow X$ be a map such that

$p(T x, T y) \leq \varphi(M(x, y))+L p^{w}(y, T x)$

for all $x, y \in X$, where $L \geq 0, M(x, y)$ as in Theorem 2 and $\varphi:[0, \infty) \rightarrow[0, \infty)$ is an upper semicontinuous from the right function such that $\varphi(t)<t$ for all $t>0$.

Then $T$ has a fixed point in $X$.

The purpose of this paper is to present a generalization of Theorem 5 which has as a particular case mappings satisfying an integral type almost contraction condition.

\section{Main results}

Let $\mathcal{F}$ be the class of functions defined by $\mathcal{F}=\{\phi:[0, \infty) \rightarrow[0, \infty): \phi$ is continuous and non decreasing $\}$.

Some examples of functions belonging to $\mathcal{F}$ are: $\phi(t)=k t$ with $k \in(0, \infty), \quad \phi(t)=\frac{t}{1+t}, \quad \phi(t)=\ln (1+t) \quad$ and $\phi(t)=\arctan t$.

Our main result is the following.

Theorem 7 Let $(X, p)$ be a 0 -complete partial metric space and $T: X \rightarrow X$ be a mapping satisfying

$\phi(p(T x, T y)) \leq \phi(\varphi(M(x, y)))+L p^{w}(y, T x)$

for all $x, y \in X$, where $L \geq 0, M(x, y)$ is defined as in Theorem $2, \phi \in \mathcal{F}$ and $\varphi$ is a $(c)$-comparison function.

Then $T$ has a fixed point $z$ in $X$ such that $p(z, z)=0$.

Proof We take $x_{0} \in X$ and consider $x_{n}=T x_{n-1}=T^{n} x_{0}$ for any $n \in \mathbb{N}$. If $x_{n}=x_{n+1}$ for some $n \in \mathbb{N}$, then $x_{n}$ is a 
fixed point of $T$ and the proof is finished. Suppose that $x_{n} \neq x_{n+1}$ for any $n \in \mathbb{N}$.

Since

$$
\begin{aligned}
& \frac{1}{2}\left[p\left(x_{n+1}, x_{n+1}\right)+p\left(x_{n}, x_{n+2}\right)\right] \leq \frac{1}{2}\left[p\left(x_{n}, x_{n+1}\right)+p\left(x_{n+1}, x_{n+2}\right)\right] \\
& \quad \leq \max \left\{p\left(x_{n}, x_{n+1}\right), p\left(x_{n+1}, x_{n+2}\right)\right\}
\end{aligned}
$$

then we have

$$
\begin{aligned}
M\left(x_{n}, x_{n+1}\right) & =\max \left\{p\left(x_{n}, x_{n+1}\right), p\left(x_{n}, x_{n+1}\right), p\left(x_{n+1}, x_{n+2}\right),\right. \\
& \left.\frac{1}{2}\left[p\left(x_{n+1}, x_{n+1}\right)+p\left(x_{n}, x_{n+2}\right)\right]\right\} \\
& =\max \left\{p\left(x_{n}, x_{n+1}\right), p\left(x_{n+1}, x_{n+2}\right)\right\} .
\end{aligned}
$$

Applying the contractive condition (2.1) we have

$$
\begin{aligned}
\phi\left(p\left(x_{n+1}, x_{n+2}\right)\right) & =\phi\left(p\left(T x_{n}, T x_{n+1}\right)\right) \\
& \leq \phi\left(\varphi\left(M\left(x_{n}, x_{n+1}\right)\right)\right)+L p^{w}\left(x_{n+1}, x_{n+1}\right) \\
& =\phi\left(\varphi\left(M\left(x_{n}, x_{n+1}\right)\right)\right)
\end{aligned}
$$

If $M\left(x_{n}, x_{n+1}\right)=p\left(x_{n+1}, x_{n+2}\right)$ for some $n \in \mathbb{N}$, then from (2.2) we obtain,

$$
\phi\left(p\left(x_{n+1}, x_{n+2}\right)\right) \leq \phi\left(\varphi\left(p\left(x_{n+1}, x_{n+2}\right)\right)\right)
$$

and, since $\phi$ is nondecreasing,

$p\left(x_{n+1}, x_{n+2}\right) \leq \varphi\left(p\left(x_{n+1}, x_{n+2}\right)\right)<p\left(x_{n+1}, x_{n+2}\right)$

which is a contradiction. Therefore $M\left(x_{n}, x_{n+1}\right)=$ $p\left(x_{n}, x_{n+1}\right)$ for all $n \in \mathbb{N}$. From (2.2), we get

$$
\phi\left(p\left(x_{n+1}, x_{n+2}\right)\right) \leq \phi\left(\varphi\left(p\left(x_{n}, x_{n+1}\right)\right)\right)
$$

and, since $\phi$ is nondecreasing,

$p\left(x_{n+1}, x_{n+2}\right) \leq \varphi\left(p\left(x_{n}, x_{n+1}\right)\right)$.

By using mathematical induction, we obtain

$p\left(x_{n+1}, x_{n+2}\right) \leq \varphi^{n+1}\left(p\left(x_{0}, x_{1}\right)\right)$.

for all $n \in \mathbb{N}$. By triangle rule, for $m>n$, we have

$$
\begin{aligned}
p\left(x_{n}, x_{m}\right) & \leq \sum_{k=n}^{m-1} p\left(x_{k}, x_{k+1}\right)-\sum_{k=n}^{m-2} p\left(x_{k+1}, x_{k+1}\right) \\
& \leq \sum_{k=n}^{m-1} p\left(x_{k}, x_{k+1}\right) \\
& \leq \sum_{k=n}^{\infty} p\left(x_{k}, x_{k+1}\right) \\
& \leq \sum_{k=n}^{\infty} \varphi^{k}\left(p\left(x_{0}, x_{1}\right)\right) .
\end{aligned}
$$

Since $\varphi$ is a (c)-comparison function, then $\sum_{k=0}^{\infty} \varphi^{k}\left(p\left(x_{0}, x_{1}\right)\right)$ is convergent and so $\left\{x_{n}\right\}$ is a 0 -Cauchy sequence in $X$. Since $X$ is 0 -complete, $\left\{x_{n}\right\}$ converges, with respect to $\tau_{p}$, to a point $z \in X$ such that

$\lim _{n \rightarrow \infty} p\left(x_{n}, z\right)=p(z, z)=0$.

Now we claim that $p(z, T z)=0$. Suppose on contrary $p(z, T z)>0$. As $\phi$ is a $(c)$-comparison function, $\phi(t)<t$ for $t>0$, As $\lim _{n \rightarrow \infty} p\left(x_{n+1}, x_{n}\right)=0$ and $\lim _{n \rightarrow \infty}$ $p\left(x_{n}, z\right)=0$, there exists $n_{0} \in \mathbb{N}$ such that for $n>n_{0}$,

$p\left(x_{n+1}, x_{n}\right)<\frac{1}{3} p(z, T z)$

and there exists $n_{1} \in \mathbb{N}$ such that for $n>n_{1}$,

$p\left(x_{n}, z\right)<\frac{1}{3} p(z, T z)$.

If we take $n>\max \left\{n_{0}, n_{1}\right\}$ then, by (2.3), (2.4) and triangular inequality, we have

$$
\begin{aligned}
\frac{1}{2}[ & \left.p\left(x_{n}, T z\right)+p\left(z, T x_{n}\right)\right] \leq \frac{1}{2}\left[p\left(x_{n}, z\right)+p(z, T z)\right. \\
& \left.-p(z, z)+p\left(z, T x_{n}\right)\right] \\
& \leq \frac{1}{2}\left[\frac{1}{3} p(z, T z)+p(z, T z)+\frac{1}{3} p(z, T z)\right] \\
& =\frac{5}{6} p(z, T z) .
\end{aligned}
$$

Now for $n>\max \left\{n_{0}, n_{1}\right\}$, then, by (2.3), (2.4) and (2.5), we have

$$
\begin{aligned}
\phi\left(p\left(x_{n+1}, T z\right)\right) & =\phi\left(p\left(T x_{n}, T z\right)\right) \\
& \leq \phi\left(\varphi\left(M\left(x_{n}, z\right)\right)+L p^{w}\left(z, x_{n+1}\right)\right. \\
& =\phi(\varphi(p(z, T z)))+L p^{w}\left(z, x_{n+1}\right) .
\end{aligned}
$$

Letting $n \rightarrow \infty$ in the last inequality, we have $\phi(p(z, T z)) \leq \phi(\varphi(p(z, T z)))$ and since $\phi$ is nondecreasing $p(z, T z) \leq \varphi(p(z, T z))<p(z, T z)$ which is a contradiction. Therefore $p(T z, z)=0$ and $z=T z$.

We can obtain the following corollaries from our main theorem.

Corollary 1 Theorem 4 .

Proof Consider $\phi=I_{[0, \infty)} \quad$ identity mapping in Theorem 7.

Notice that if $f:[0, \infty) \rightarrow[0, \infty)$ is a Lebesgue-integrable mapping then the function defined by

$\phi(t)=\int_{0}^{t} f(s) d s \quad$ fort $\in[0, \infty)$,

belongs to $\mathcal{F}$. Therefore we can obtain the following corollary. 
Corollary 2 Let $(X, p)$ be a 0-complete partial metric space and $T: X \rightarrow X$ be a mapping satisfying

$$
\int_{0}^{p(T x, T y)} f(s) d s \leq \int_{0}^{\varphi(M(x, y))} f(s) d s+L p^{w}(y, T x)
$$

for all $x, y \in X$, where $L \geq 0, M(x, y)$ as in Theorem $2, \varphi$ is a $(c)$-comparison function and $f:[0, \infty) \rightarrow[0, \infty)$ is a Lebesgue-integrable mapping.

Then $T$ has a fixed point in $X$.

Now we give an illustrative example.

Example 4 Let $X=A \cup B$, where $A=\{0\} \cup\left\{\frac{1}{n}: n \in\right.$ $\{1,2, \ldots\}\}, B=\{2,3,4, \ldots\}$ and

$p(x, y)=\left\{\begin{array}{ll}\max \{x, y\}, & x \neq y \\ 0, & x=y\end{array}\right.$.

Then $(X, p)$ is a partial metric space and it is also 0-complete. Define $T: X \rightarrow X$ by

$$
T x=\left\{\begin{array}{ll}
x^{3}, & x \in A \\
\frac{1}{x}, & x \in B
\end{array} .\right.
$$

We show that the contractive condition (2.1) of Theorem 7 is satisfied for $\phi(t)=t, \varphi(t)=\frac{t}{2}$ and $L=2$.

Now consider the following cases.

Case 1. If $x=y$, then $p(T x, T y)=0$ and so the result is clear. Therefore we will assume $x \neq y$ in the following cases.

Case 2. Let $x, y \in A$. Then (note that if $x=1$ or $y=1$ then $\inf \left\{\left|y-x^{3}\right|: x, y \in A\right.$ with $\left.x \neq y\right\}=\frac{1}{2}$. If $x \neq 1$ and $y \neq 1$, then $\left.x^{3} \leq \frac{1}{2} x\right)$

$$
\begin{aligned}
p(T x, T y) & =\max \left\{x^{3}, y^{3}\right\} \\
& \leq \frac{1}{2} \max \{x, y\}+2\left|y-x^{3}\right| \\
& =\frac{1}{2} p(x, y)+2 p^{w}(y, T x) \\
& =\varphi(M(x, y))+L p^{w}(y, T x) .
\end{aligned}
$$

Case 3. Let $x, y \in B$. Then

$$
\begin{aligned}
p(T x, T y) & =\max \left\{\frac{1}{x}, \frac{1}{y}\right\} \\
& =\frac{1}{\min \{x, y\}} \\
& \leq \frac{1}{2} \max \{x, y\} \\
& =\frac{1}{2} p(x, y) \\
& \leq \varphi(M(x, y))+L p^{w}(y, T x) .
\end{aligned}
$$

Case 4. Let $x \in A$ and $y \in B$. Then

$$
\begin{aligned}
p(T x, T y) & =\max \left\{x^{3}, \frac{1}{y}\right\} \\
& \leq \frac{1}{2} y \\
& =\frac{1}{2} p(x, y) \\
& \leq \varphi(M(x, y))+L p^{w}(y, T x) .
\end{aligned}
$$

Case 5. Let $x \in B$ and $y \in A$. This case is similar to Case 4 . Hence, all conditions of Theorem 7 are satisfied. Therefore $T$ has a fixed point in $X$.

Note that $p(T 0, T 1)=1=M(0,1)$, then the condition of (1.1) is not satisfied, because we can not find a function $\varphi$ satisfying

$p(T 0, T 1)=1 \leq \varphi(M(0,1))=\varphi(1)$

and the condition (1) or (2) of Theorem 2. Therefore Theorem 2 is not applicable to this example.

In the above, we show that if $T$ is a generalized almost contraction then it has a fixed point. But in order to guarantee the uniqueness of the fixed point of $T$, we have to consider an additional condition, as in the following theorem.

Theorem 8 Let $(X, p)$ be a 0-complete partial metric space and $T: X \rightarrow X$ be a map such that (2.1) holds. Suppose $T$ also satisfies the following condition: there exists a comparison function $\varphi_{1}$, some $L_{1} \geq 0$ and $\phi_{1} \in \mathcal{F}$ with $\phi_{1}(t)>0$ for $t>0$ such that

$$
\phi_{1}(p(T x, T y)) \leq \phi_{1}\left(\varphi_{1}(M(x, y))\right)+L_{1} p^{w}(x, T x)
$$

holds, for all $x, y \in X$. Then $T$ has a unique fixed point in $X$.

Proof Suppose that, there are two fixed points $z$ and $w$ of $T$. If $p(z, w)=0$, it is clear that $z=w$. Assume that $p(z, w)>0$. By (2.6) with $x=z$ and $y=w$, we have

$$
\begin{aligned}
0 & <\phi_{1}(p(z, w))=\phi_{1}(p(T z, T w)) \\
& \leq \phi_{1}\left(\varphi_{1}(M(z, w))\right)+L_{1} p^{w}(z, T z) \\
& =\phi_{1}\left(\varphi_{1}(M(z, w))\right) \\
& =\phi_{1}\left(\varphi_{1}(p(z, w))\right)
\end{aligned}
$$

since $\phi$ is nondecreasing

$$
0<p(z, w) \leq \varphi_{1}(p(z, w))<p(z, w)
$$

which is a contradiction. Therefore $T$ has a unique fixed point.

Acknowledgments The authors would like to thank the referees for their helpful advice which led them to present this paper.

Open Access This article is distributed under the terms of the Creative Commons Attribution License which permits any use, distribution, and reproduction in any medium, provided the original author(s) and the source are credited. 


\section{References}

1. Abdeljawad, T., Karapinar, E., Tas, K.: Existence and uniqueness of a common fixed point on partial metric spaces. Appl. Math. Lett. 24, 1900-1904 (2011)

2. Acar, Ö., Altun, I.: Some generalizations of Caristi type fixed point theorem on partial metric spaces. Filomat 26(4), 833-837 (2012)

3. Acar, Ö., Altun, I., Romaguera, S.: Caristi's type mappings on complete partial metric space. Fixed Point Theory (Cluj-Napoca) 14(1), 3-10 (2013)

4. Acar, Ö., Berinde, V., Altun, I.: Fixed point theorems for Ciric strong almost contractions in partial metric spaces. J. Fixed Point Theory Appl. 12, 247-259 (2012)

5. Altun, I., Acar, Ö.: Fixed point theorems for weak contractions in the sense of Berinde on partial metric spaces. Topol. Appl. 159, 2642-2648 (2012)

6. Altun, I., Erduran, A.: Fixed point theorems for monotone mappings on partial metric spaces. Fixed Point Theory Appl. pp. 10 (2011). Article ID 508730

7. Altun, I., Romaguera, S.: Characterizations of partial metric completeness in terms of weakly contractive mappings having fixed point. Appl. Anal. Discrete Math. 6, 247-256 (2012)

8. Altun, I., Sola, F., Simsek, H.: Generalized contractions on partial metric spaces. Topol. Appl. 157, 2778-2785 (2010)

9. Berinde, V.: Approximating fixed points of weak contractions using the Picard iteration. Nonlinear Anal. Forum 9(1), 43-53 (2004)

10. Berinde, V.: On the approximation of fixed points of weak contractive mappings. Carpath. J. Math. 19(1), 7-22 (2003)

11. Berinde, V.: Iterative Approximation of Fixed Points. Springer, Berlin (2007)

12. Berinde, V.: Approximating fixed points of weak $\varphi$-contractions using the picard iteration. Fixed Point Theory, pp. 131-147 (2003)

13. Berinde, V.: Some remarks on a fixed point theorem for Ćirićtype almost contractions. Carpath. J. Math. 25(2), 157-162 (2009)
14. Ćirić, L., Samet, B., Aydi, H., Vetro, C.: Common fixed points of generalized contractions on partial metric spaces and an application. Appl. Math. Comput. 218, 2398-2406 (2011)

15. Cobzaş, S.: Completeness in quasi-metric spaces and Ekeland variational principle. Topol. Appl. 158, 1073-1084 (2011)

16. Escardo, M.H.: Pcf extended with real numbers. Theor. Comput. Sci. 162, 79-115 (1996)

17. Heckmann, R.: Approximation of metric spaces by partial metric spaces. Appl. Categ. Struct. 7, 71-83 (1999)

18. Ilic, D., Pavlovic, V., Rakocevic, V.: Some new extensions of Banach's contraction principle to partial metric space. Appl. Math. Lett. 24, 1326-1330 (2011)

19. Karapinar, E., Erhan, I.M.: Fixed point theorems for operators on partial metric spaces. Appl. Math. Lett. 24, 1894-1899 (2011)

20. Matthews, S.G.: Partial metric topology. In: Proceedings of 8th Summer Conference on General Topology and Applications, Annals of the New York Academy of Sciences vol. 728, pp. 183-197 (1994)

21. Oltra, S., Valero, O.: Banach's fixed point theorem for partial metric spaces. Rend. Istid. Math. Univ. Trieste 36, 17-26 (2004)

22. Romaguera, S.: A Kirk type characterization of completeness for partial metric spaces. Fixed Point Theory Appl. pp. 6 (2010). Article ID 493298

23. Romaguera, S.: Fixed point theorems for generalized contractions on partial metric spaces. Topol. Appl. 218, 2398-2406 (2011)

24. Romaguera, S.: Matkowski's type theorems for generalized contractions on (ordered) partial metric spaces. Appl. Gen. Topol. 12, 213-220 (2011)

25. Samet, B., Rajović, M., Lazović, R., Stojiljković, R.: Common fixed-point results for nonlinear contractions in ordered partial metric spaces. Fixed Point Theory Appl. 2011:71 (2011)

26. Shatanawi, W., Samet, B., Abbas, M.: Coupled fixed point theorems for mixed monotone mappings in ordered partial metric spaces. Math. Comput. Model. 55, 680-687 (2012)

27. Valero, O.: On Banach fixed point theorems for partial metric spaces. Appl. Gen. Topol. 6, 229-240 (2005) 